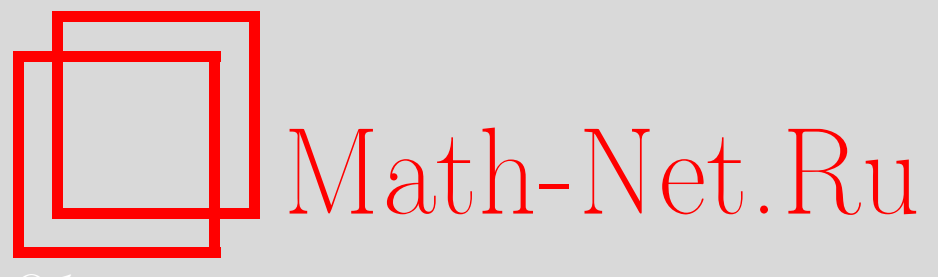

А. Е. Кирнасов, Установочные эксперименты для автоматов с изменяемой логикой поведения, Дискрет. матем., 2005, том 17, выпуск 2, 102-116

DOI: https://doi.org/10.4213/dm102

Использование Общероссийского математического портала Math-Net.Ru подразумевает, что вы прочитали и согласны с пользовательским соглашением http: //www . mathnet.ru/rus/agreement

Параметры загрузки:

IP : 54.174 .149 .18

26 апреля 2023 г., 15:02:04

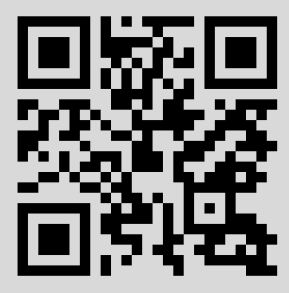




\title{
Установочные эксперименты для автоматов с изменяемой логикой поведения
}

๑) 2005 г. А. Е. Кирнасов

\begin{abstract}
В статье рассматривается задача о зависимости длины простого условного установочного эксперимента автомата от локальных преобразований диаграммы переходов и выходов автомата. Приводятся верхние и нижние оценки для функции Шеннона длины эксперимента при возможности произвольно перенаправлять любые $p$ стрелок в диаграмме Мура автомата, а также изменять значение функции выходов в $p$ точках.
\end{abstract}

\section{1. Введение}

Пусть $\mathfrak{U}=(A, Q, B, \varphi, \psi)-$ автомат, где $A-$ входной алфавит, $B$ - выходной алфавит, $Q-$ множество состояний, $\varphi-$ функщия переходов, $\psi-$ функция выходов. Мы также будем рассматривать естественные продолжения функций $\varphi$ и $\psi$ на слова из алфавита $A$, которые также будем обозначать $\varphi$ и $\psi$. Обозначим $K(\mathfrak{U}, p)$ класс автоматов, диаграмма Мура которых отличается от диаграммы Мура автомата $A$ переключением произвольных $p$ стрелок. Обозначим также $K_{1}(\mathfrak{A}, p)$ класс автоматов, получающихся из автомата $\mathfrak{U}$ путем изменения функщии выходов в $p$ точках. Более точно, предполагается, что имеется бесконечный выходной алфавит $B^{\prime}$, включающий в себя выходной алфавит $B$ автомата $\mathfrak{U}$ в качестве подмножества. Разрешается для произвольных $p$ точек множества $Q$ поменять значение функиии выходов произвольным образом. Обозначим $L(\mathfrak{U}, p)=\min L(\mathfrak{U})$, где $L(\mathfrak{U})$ - длина простого условного установочного эксперимента автомата $\mathfrak{A}$ и минимум берется по всем автоматам из класса $K(\mathfrak{U}, p)$. Обозначим также $L^{\prime}(\mathfrak{U}, p)=\min L(\mathfrak{U})$, где минимум берется по всем автоматам $\mathfrak{U}$ из класса $K_{1}(\mathfrak{U}, p)$. Вопросы о зависимости свойств экспериментов от преобразований диаграммы автомата рассматривались в [3]. В этой работе рассматривался вопрос о нахождении оценок шенноновского типа длины контрольного эксперимента с автоматом [1] в так называемом локально-порожденном классе автомата [3].

Степенью отличимости автомата называется максимальная из кратчайших длин слов, отличающих пары состояний автомата [1].

Во втором параграфе вводятся основные понятия и формулируются основные результаты. В третьем параграфе вводятся вспомогательные понятия. В четвертом параграфе исследуется связь длины простого условного установочного эксперимента со степенью отличимости автомата. В пятом параграфе исследуется понятие условной степени отличимости автомата. В шестом и седьмом параграфах исследуется зависимость степени отличимости автомата от локальных преобразований диаграммы Мура автомата и функции его переходов. В восьмом параграфе доказываются верхние и нижние оценки для 
определяемых ниже функций $L(n, p)$ и $L^{\prime}(n, p)$ в общем случае и для специально определенного класса автоматов-идентификаторов.

\section{2. Основные определения и результаты}

Пусть дан автомат $\mathfrak{U}$. Обозначим через $h(\mathfrak{U})$ степень отличимости автомата $\mathfrak{U}$, а через $L(\mathfrak{U})$ длину простого условного установочного эксперимента автомата $\mathfrak{U}$.

Введем также понятие остаточной степени отличимости автомата $\mathfrak{U}$. Предположим, что для любых двух состояний автомата $q_{1}, q_{2}$ автомата $\mathfrak{Q}$ выполняется одно из условий:

(1) существует слово $\alpha$ такое, что его длина $l(\alpha) \leqslant h$ и $\psi\left(q_{1}, \alpha\right) \neq \psi\left(q_{2}, \alpha\right)$,

(2) существует слово $\alpha$ такое, что $l(\alpha) \leqslant h$ и $\varphi\left(q_{1}, \alpha\right)=\varphi\left(q_{2}, \alpha\right)$.

Пусть $h$ - минимальное число с таким свойством. В таком случае скажем, что остаточная степень отличимости автомата $\mathfrak{U}$ есть $h$ и будем писать $h_{\text {fin }}(\mathfrak{U})=h$.

Введем понятие $R_{2}$-неполного автомата. Пусть дан автомат $\mathfrak{U}=(A, Q, B, \varphi, \psi)$ и его степень отличимости равна $h$. Пусть $\left\{R_{k}, 0 \leqslant k \leqslant h\right\}$ - семейство отношений эквивалентности на множестве $Q$ состояний автомата, отношений неотличимости словами длины $k$. Занумеруем $R_{1}$-компоненты эквивалентных состояний автомата числами от 1 до $k$. Таким образом,

$$
Q=\bigcup_{1 \leqslant i \leqslant k} Q_{i},
$$

где $Q_{i}$ - различные $R_{1}$-компоненты автомата $\mathfrak{X}$.

Для каждого состояния $q \in Q$ определим отображение $f_{q}: A \rightarrow\{1, \ldots, k\}$ следующим образом: $f_{q}(a)=i$, если $\varphi(q, a) \in Q_{i}, i=1, \ldots, k$. Автомат назовем $R_{2}$-неполным, если найдется отображение $f: A \rightarrow\{1, \ldots, k\}$ такое, что $f \neq f_{q}$ для всех $q \in Q$.

Для автомата $\mathfrak{U}=(A, Q, B, \varphi, \psi)$ обозначим $K(\mathfrak{U}, p)$ класс автоматов

$$
\mathfrak{U}^{\prime}=\left(A, Q, B, \varphi^{\prime}, \psi\right)
$$

таких, что существует не более $p$ пар $(q, a)$, для которых $\varphi(q, a) \neq \varphi^{\prime}(q, a)$.

Для автомата $\mathfrak{U}=(A, Q, B, \varphi, \psi)$ обозначим также $K^{\prime}(\mathfrak{U}, p)$ следующий класс автоматов. Рассмотрим произвольный бесконечный алфавит $B^{\prime}$, содержащий алфавит $B$ в качестве подмножества. В класс автоматов $K^{\prime}$ включаются все те и только те автоматы $\mathfrak{U}^{\prime}=\left(A, Q, B^{\prime \prime}, \varphi, \psi^{\prime}\right)$ с $B^{\prime \prime} \subset B^{\prime}$ и $B \subseteq B^{\prime \prime}$ такие, что существует не более $p$ пар $(q, a)$, для которых $\psi(q, a) \neq \psi^{\prime}(q, a)$.

Положим $h(\mathfrak{U}, p)=\min h_{\text {fin }}(\mathfrak{B})$, где минимум берется по всем автоматам $\mathfrak{B}$ из класса $K(\mathfrak{U}, p)$. Положим также $h^{\prime}(\mathfrak{U}, p)=\min h(\mathfrak{B})$, где минимум берется по всем автоматам $\mathfrak{B}$ из класса $K^{\prime}(\mathfrak{A}, p)$.

Рассмотрим функцию $h(n, m, p)=\max h(\mathfrak{A}, p)$, где максимум берется в классе $R_{2}$-неполных автоматов с $n$ состояниями и $m$ входными символами. Аналогично введем функцию $h^{\prime}(n, p)=\max h(\mathfrak{U}, p)$, где максимум берется в классе всех автоматов $\mathfrak{U}$ с $n$ состояниями.

Введем понятие класса автоматов-идентификаторов. Автомат $\mathfrak{U}=(A, Q, B, \varphi, \psi)$ назовем идентификатором, если найдется такое состояние $q_{0}$ автомата $\mathfrak{A}$, что для всех $q \neq q_{0}$ в $Q$ существует $\alpha$ такое, что $\varphi(q, \alpha)=q_{0}$ и существуют $b_{0}, b_{1} \in B$ такие, что $b_{0} \neq b_{1}$ и для всех $q \neq q_{0}$ и всех $a \in A$ выполняется равенство $\psi(q, a)=b_{0}$ и найдется $a_{0} \in A$ такое, что $\psi\left(q_{0}, a_{0}\right)=b_{1}$. 
Пусть $h_{\mathrm{id}}(n, p)=\max h(\mathfrak{U}, p)$, где максимум берется по всем автоматам-идентификаторам с $n$ состояниями и аналогично $h_{\mathrm{id}}^{\prime}(n, p)=\max h^{\prime}(\mathscr{U}, p)$, где максимум берется по всем автоматам-идентификаторам с $n$ состояниями.

Положим $L(n, m, p)=\max L(\mathfrak{U}, p)$, где максимум берется по $R_{2}$-неполным автоматам с $n$ состояниями и $m$ входными символами. Положим также $L^{\prime}(n, p)=\max L^{\prime}(\mathfrak{U}, p)$, где максимум берется по классу автоматов с $n$ состояниями, и $L_{\text {id }}(n, p)=\max L(\mathfrak{A}, p)$, где максимум берется по классу автоматов-идентификаторов с $\boldsymbol{n}$ состояниями. Наконец, пусть $L_{\text {id }}^{\prime}(n, p)=\max L^{\prime}(\mathfrak{U}, p)$, где максимум берется в классе автоматов-идентификаторов с $n$ состояниями.

Введем также функцию двух переменных

$$
g(n, p)= \begin{cases}{[(n-1) /(p+1)]+1,} & \text { если } n-1 \text { не делится нацело на } p+1, \\ (n-1) /(p+1) & \text { в противном случае. }\end{cases}
$$

Сформулируем теперь основные результаты.

Теорема 1. При $n \rightarrow \infty u$ фиксированных $m u p$

$$
h(n, m, p) \asymp n, \quad h^{\prime}(n, p) \asymp n .
$$

Теорема 2. При $n, p \rightarrow \infty$

$$
h_{\mathrm{id}}(n, p) \sim n / p, \quad h_{\mathrm{id}}^{\prime}(n, p) \sim n / p .
$$

Теорема 3. При $n \rightarrow \infty u$ фиксированных $m u p$

$$
L(n, m, p) \asymp n^{2}, \quad L^{\prime}(n, p) \asymp n^{2} .
$$

Теорема 4. При $n, p \rightarrow \infty$

$$
L_{\text {id }}(n, p) \asymp n^{2} / p, \quad L_{\text {id }}^{\prime}(n, p) \asymp n^{2} / p .
$$

\section{3. Вспомогательные понятия и результаты}

Введем понятие условной степени отличимости автомата порядка $p$. Пусть $R$ - отношение эквивалентности на некотором множестве $Q$. Множество классов эквивалентности отношения $R$ образует некоторое разбиение множества $Q$, которое будем обозначать $T(R)$. Введем понятие порядка и частичного порядка подразбиения $T^{\prime}$ относительно разбиения $T$. Пусть $T$ - разбиение множества $Q$, а $T^{\prime}$ - его подразбиение. Пусть $Q_{0}, Q_{1}, \ldots, Q_{i}$ - те элементы разбиения $T$, которые подразбиваются в подразбиении $T^{\prime}$. Эти элементы назовем базовыми элементами разбиения $T$ относительно подразбиения $T^{\prime}$. Представим эти элементы следующим образом:

$$
Q_{0}=\bigcup_{1 \leqslant t \leqslant r_{0}} Q_{0, t}, \quad Q_{1}=\bigcup_{1 \leqslant t \leqslant r_{1}} Q_{1, t}, \ldots, \quad Q_{i}=\bigcup_{1 \leqslant t \leqslant r_{i}} Q_{i, t} .
$$

Введем обозначение $g_{q, s}=\left|Q_{q, s}\right|$. Также положим $g_{j}=\left|Q_{j}\right|$. Рассмотрим также величину $G_{j}=\min _{1 \leqslant t \leqslant r_{j}}\left(g_{j}-g_{j, t}\right)$. Предположим, что указанный минимум достигается при $t=t_{j}$. 
Порядком подразбиения $T^{\prime}$ относительно разбиения $T$, обозначаемым $\operatorname{ord}\left(T^{\prime}, T\right)$, назовем величину

$$
\operatorname{ord}\left(T^{\prime}, T\right)=\sum_{j=0}^{i} G_{j}
$$

Пусть теперь дан автомат $\mathfrak{A}=(A, Q, B, \varphi, \psi)$. Пусть также его степень отличимости есть $h$. Рассмотрим произвольную монотонную последовательность $\alpha$ вида

$$
0=k_{0}<k_{1}<\ldots<k_{s}=h .
$$

Класс таких последовательностей обозначим $\mathrm{Mon}_{h}$. Порядком дробления автомата $\mathfrak{A}$ относительно последовательности $\alpha$, обозначаемым $\operatorname{fr}(\mathfrak{U}, \alpha)$, назовем величину

$$
\operatorname{fr}(\mathfrak{A}, \alpha)=\sum_{1 \leqslant j \leqslant s} \operatorname{ord}\left(T\left(R_{k_{j}}\right), T\left(R_{k_{j}-1}\right)\right) .
$$

Назовем также дисперсией последовательности $\alpha$ величину

$$
\operatorname{disp}(\alpha)=\max _{1 \leqslant i \leqslant s}\left(k_{i}-k_{i-1}\right)
$$

Наконец, определим условную степень отличимости автомата порядка $p$ равенством

$$
h_{c}(\mathfrak{U}, p)=\min _{\alpha \in \operatorname{Mon}_{h}, \text { fr( }(\mathscr{A}, \alpha) \leqslant p} \operatorname{disp}(\alpha) .
$$

Сформулируем и докажем следующие две теоремы, которые будут использоваться в дальнейшем.

Теорема 5. Для автомата $\mathfrak{U}$ с условной степенью отличимости порядка $p$, равной $h_{0}$, найдется автомат $\mathfrak{B} \in K^{\prime}(\mathfrak{U}, p)$ такой, что $h(\mathfrak{B}) \leqslant h_{0}$.

Доказательство. Зафиксируем произвольный бесконечный алфавит $B^{\prime}$ такой, что $B \subseteq B^{\prime}$. По определению найдется последовательность $\alpha \in \operatorname{Mon}_{h} \mathrm{c} \operatorname{disp}(\alpha)=h_{0}$ такая, что $\operatorname{fr}(\mathfrak{U}, \alpha) \leqslant p$. Рассмотрим разбиение $T_{j}=T\left(R_{k_{j}-1}\right)$ и его подразбиение $T_{j}^{\prime}=T\left(R_{k_{j}}\right)$ для $1 \leqslant j \leqslant s$. Пусть $Q_{j, 1}, Q_{j, 2}, \ldots, Q_{j, r_{j}}-$ базовые элементы разбиения $T_{j}$ относительно подразбиения $T_{j}^{\prime}$. Рассмотрим множество $Q_{j, i}$. Представим его как объединение

$$
Q_{j, i}=\bigcup_{i \leqslant t \leqslant r_{j, i}} Q_{j, i, t}
$$

Пусть, как и выше, $g(j, i, t)=\left|Q_{j, i, t}\right|$. Положим также

$$
G(j, i)=\sum_{1 \leqslant t \leqslant r_{j, i}} g(j, i, t)
$$

Наконец, пусть $t_{0, j, i}-$ значение параметра $t$, при котором достигается минимум выражения $G(j, i)-g(j, i, t)$. Рассмотрим теперь следующее семейство разбиений множества $Q$ :

$$
\mathcal{R}=\left\{\tau_{j}=\left\{Q_{j, i, t} \mid 1 \leqslant i \leqslant r_{j}, 1 \leqslant t \leqslant r_{j, i, t} \neq t_{0, j, i}, Q \backslash \bigcup_{1 \leqslant t \leqslant r_{j, i}, t \neq t_{0, j, i}} Q_{j, i, t}\right\}\right\} .
$$


Рассмотрим теперь разбиение

$$
\tau=\bigcap_{1 \leqslant j \leqslant s} \tau_{j}
$$

Представим разбиение $₹$ в виде

$$
\mathfrak{T}=\left\{V_{n} \mid l \leqslant n \leqslant N\right\} .
$$

Нетрудно видеть, что существует ровно одно значение $n=n_{0}$, для которого $V_{n_{0}} \cap Q_{j, i, t}=\varnothing$ для всех $j, i, t 1 \leqslant i \leqslant t, 1 \leqslant i \leqslant r_{j}, 1 \leqslant t \leqslant r_{j, i, t} \neq t_{0, j, i}$. Отметим следующий факт. Предположим, что имеются два разбиения множества $Q$,

$$
T_{1}=\left\{V_{i} \mid 1 \leqslant i \leqslant k\right\}, \quad T_{2}=\left\{W_{j} \mid 1 \leqslant j \leqslant l\right\} .
$$

Пусть также в каждом из этих разбиений выделены элементы $V_{i_{0}}$ и $W_{i_{0}}$. Допустим, что для всех $j \neq j_{0}$ существует $i(j)$ такое, что $W_{j} \in V_{i(j)}$. В таком случае будем говорить, что разбиение $T_{1}$ доминирует разбиение $T_{2}$. Пусть $T=T_{1} \cap T_{2}$. Представим это пересечение в виде $T=\left\{S_{q} \mid 1 \leqslant q \leqslant m\right\}$. Тогда нетрудно понять, что найдется $q_{0}$ такое, что $S_{q_{0}} \cap V_{i}=\varnothing$ и $S_{q_{0}} \cap W_{j}=\varnothing$ для всех $i \neq i_{0}, j \neq j_{0}$. Пусть

$$
\sum_{1 \leqslant i \leqslant k, i \neq i_{0}}\left|V_{i}\right|=m, \quad \sum_{1 \leqslant j \leqslant l, j \neq j_{0}}\left|W_{j}\right|=n .
$$

Тогда

$$
\sum_{1 \leqslant q \leqslant m, q \neq q_{0}}\left|S_{q}\right|=m+n .
$$

Очевидно, что для всех $1 \leqslant i<j \leqslant s$ разбиение $\mathfrak{\Upsilon}_{i}$ доминирует разбиение $\mathfrak{\Sigma}_{j}$. Отсюда и указанного свойства по индукщии нетрудно заключить, что

$$
\sum_{V \in \mathcal{T}, V \neq V_{n_{0}}}|V| \leqslant p .
$$

Выберем произвольно символ $а$ из входного алфавита $A$ автомата $\mathfrak{\mathscr { A }}$. Положим

$$
B^{\prime \prime}=B^{\prime} \cup \bigcup_{1 \leqslant n \leqslant N, n \neq n_{0}} b_{n}
$$

Также положим $\psi^{\prime}(q, a)=b_{n}$, если $q \in V_{n}$. Для всех пар $\left(q, a^{\prime}\right)$ таких, что либо $a \neq a^{\prime}$, либо $q \in V_{n_{0}}$, положим $\psi^{\prime}(q, a)=\psi(q, a)$. Теперь рассмотрим автомат $\mathfrak{B}=\left(A, Q, B^{\prime \prime}, \varphi, \psi^{\prime}\right)$. Очевидно, что $\mathfrak{B} \in K^{\prime}(\mathfrak{A}, p)$. Покажем, что $h(\mathfrak{B}) \leqslant h_{0}$.

Рассмотрим два произвольных состояния $q_{0}, q_{1} \in Q$. Предположим, что кратчайшее слово, которое их различает, есть $\beta$ и имеет длину $k$. Найдется такое $j$, что $k_{j} \leqslant k<k_{j+1}$. Пусть $\beta_{0}$ - начальный отрезок слова $\beta$ длины $k-k_{j}$. В случае, когда $k=k_{j}$, полагаем, что $\beta_{0}$ - пустое слово. Рассмотрим состояния $q_{0}^{\prime}=\varphi\left(q_{0}, \beta_{0}\right)$ и $q_{1}^{\prime}=\varphi\left(q_{1}, \beta_{0}\right)$. Очевидно, что состояния $q_{0}^{\prime}, q_{1}^{\prime}$ лежат в одном классе эквивалентности отношения $R_{k_{j}-1}$, но в разных классах эквивалентности отношения $R_{k_{j}}$. Отсюда следует, что $q_{0}^{\prime} \in Q_{j, i, t_{0}}$, а $q_{1}^{\prime} \in Q_{j, i, t_{1}}$ при некотором $i$ и некоторых $t_{0} \neq t_{1}$, а значит, по построению разбиения $\mathfrak{T}$ найдутся два различных элемента $V_{n_{1}}$ и $V_{n_{2}}$ этого разбиения такие, 
что $q_{0}^{\prime} \in V_{n_{1}}$ и $q_{1}^{\prime} \in V_{n_{2}}$. Следовательно, по построению автомата $\mathfrak{B}$ состояния $q_{0}^{\prime}$ и $q_{1}^{\prime}$ находятся в разных классах эквивалентности отношения $R_{1}$. Пусть $\psi^{\prime}\left(q_{0}^{\prime}, a_{0}\right) \neq \psi^{\prime}\left(q_{1}^{\prime}, a_{0}\right)$. Введя обозначение $\gamma=\beta_{0} *\left\{\alpha_{0}\right\}$, где $*$ означает конкатенацию слов, получим, что $\psi^{\prime}\left(q_{0}, \gamma\right) \neq \psi^{\prime}\left(q_{1}, \gamma\right)$. Очевидно, что длина слова $\gamma$ не превосходит $h_{0}$, следовательно, в автомате $\mathfrak{B}$ состояния $q_{0}$ и $q_{1}$ отличимы некоторым словом длины, меньшей $h_{0}$. Отсюда следует, что $h(\mathfrak{B}) \leqslant h_{0}$. Теорема доказана.

Теорема 6. Если условная степень отличимости автомата $\mathfrak{X}$ порядка $p$, равна $h_{0}$, автомат $\mathfrak{X}$ является $R_{2}$-неполным $u|A|=m$, то найдется автомат $\mathfrak{B} \in K(\mathfrak{U}, m * p)$ такой, что $h_{\text {fin }}(\mathfrak{B}) \leqslant h_{0}+1$.

Доказательство. Первая часть доказательства совпадает с первой частью доказательст-

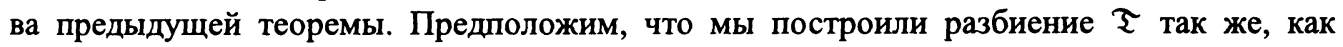
при доказательстве предыдущей теоремы. Зафиксируем отображение $f$, участвующее в определении $R_{2}$-неполного автомата. В каждой $R_{1}$-компоненте $Q_{i}$ автомата $\mathfrak{U}$ выберем по состоянию $q_{i}$. Пусть $q \in V_{i}, i \neq n_{0}$. Для произвольного $a \in A$ положим $\varphi^{\prime}(q, a)=q_{f(a)}$. Во всех остальных случаях положим $\varphi^{\prime}(q, a)=\varphi(q, a)$. Теперь рассмотрим автомат $\mathfrak{B}=\left(A, Q, B, \varphi^{\prime}, \psi\right)$. Очевидно, что $\mathfrak{B} \in K^{\prime}(\mathfrak{U}, m * p)$. Покажем, что $h_{\text {fin }}(\mathfrak{B}) \leqslant h_{0}+1$.

Рассмотрим два произвольных состояния $q_{0}, q_{1} \in Q$. Предположим, что кратчайшее слово $\beta$, которое их различает в автомате $\mathfrak{U}$, имеет длину $k$. Найдется такое $j$, что $k_{j} \leqslant k<k_{j+i}$. Пусть $\beta_{0}-$ начальный отрезок слова $\beta$ длины $k-k_{j}$. В случае, когда $k=k_{j}$, полагаем, что $\beta_{0}$ - пустое слово. Положим $r=k-k_{j}$. Для всех $j, 0 \leqslant j \leqslant r$, положим $g_{0, j}=\varphi\left(q_{0}, \beta_{i}\right), g_{1, j}=\varphi\left(q_{1}, \beta_{i}\right)$, где $\beta_{i}$ - начальный отрезок слова $\beta_{0}$ длины $j$. Пусть $j_{0}-$ минимальное значение $j$ такое, что либо $q_{0, j_{0}} \in V_{n_{0}}, q_{1, j_{0}} \notin V_{n_{0}}$, либо $q_{0, j_{0}} \notin V_{n_{0}}, q_{1, j_{0}} \in V_{n_{0}}$, либо $q_{0, j_{0}} \notin V_{n_{0}}, q_{1, j_{0}} \notin V_{n_{0}}$. Исходя из определения разбиения $₹$ видим, что такое $j_{0} \leqslant r$ найдется. Рассмотрим состояния $q_{0}^{\prime}=\varphi\left(q_{0}, \beta_{j_{0}}\right)$ и $q_{1}^{\prime}=\varphi\left(q_{1}, \beta_{j_{0}}\right)$. В первом случае найдется символ $a \in A$ такой, что $\varphi^{\prime}\left(q_{0}^{\prime}, a\right) \in Q_{s_{1}}, \varphi^{\prime}\left(q_{1}^{\prime}, a\right) \in Q_{s_{2}}$, где $Q_{s_{1}}$ и $Q_{s_{1}}$ - две различные $R_{1}$-компоненты автомата $\mathfrak{U}$. Следовательно, найдется слово длины, не превосходящей $h_{0}+1$, которое в автомате $\mathfrak{B}$ остаточно отличит состояния $q_{0}, q_{1}$. Точно так же разбирается второй случай. Наконец, в третьем случае $\varphi^{\prime}\left(q_{0}^{\prime}, a\right)=\varphi^{\prime}\left(q_{1}^{\prime}, a\right)$ для всех $a \in A$, следовательно, найдется слово длины, не большей $h_{0}$, остаточно отличающее состояния $q_{0}$ и $q_{1}$ в автомате $\mathfrak{B}$. Это заканчивает доказательство того, что $h_{\text {fin }} \mathfrak{B} \leqslant h_{0}+1$.

\section{4. Теоремы о связи степени отличимости автомата с длиной установочного эксперимента}

Сформулируем и докажем следующую теорему.

Теорема 7. Для автомата $\mathfrak{U}$ степени отличимости $h$ с п состояниями

$$
L(\mathfrak{U}) \leqslant \frac{1}{2} h(2 n-1-h)
$$

и эта оченка достижима.

Доказательство. Рассмотрим автомат $\mathfrak{U}=(A, Q, B, \varphi, \psi)$ с $|Q|=n$ и степенью отличимости $h$. Получим верхнюю оценку для длины простого условного установочного эксперимента $l(\mathfrak{U})$. Рассмотрим семейство отношений $R_{k}$ на множестве $Q$, где $R_{k}-$ 
отношение неотличимости состояний словами длины $k$. Обозначим $\xi_{s}$ мощность максимального из классов эквивалентности отношения $R_{s}$. Очевидно, что $\xi_{h}=1, \xi_{0}=n$ и $\xi_{i+1} \leqslant \xi_{i}$. Выделим из последовательности $\xi_{n}$ монотонную подпоследовательность $\xi_{n_{k}}$. Очевидно, что $n_{0}=0, n_{1}=1$. Пусть $h=n_{k_{0}}$. Докажем, что

$$
L(\mathfrak{X}) \leqslant \sum_{j=1}^{k_{0}} n_{j}\left(\xi_{n_{j}-1}-\xi_{n_{j}}\right) .
$$

Построим дерево простого условного установочного эксперимента следующим образом. Пусть уже построено дерево $T$, удовлетворяющее всем свойствам условного установочного эксперимента кроме одного: некоторым листьям приписаны подмножества множества $Q$, содержащие более одного элемента. Рассмотрим один из таких листьев. Пусть ему приписано множество $Q^{\prime}$. Определим $k$ как минимальное натуральное число такое, что существуют $q_{1}, q_{2} \in Q^{\prime}$, которые не являются $R_{k}$-эквивалентными. Тогда припишем листу слово $\alpha$ длины $k$ такое, что $\psi\left(q_{1}, \alpha\right) \neq \psi\left(q_{2}, \alpha\right)$, и представим $Q^{\prime}$ в виде $Q^{\prime}=\bigcup_{i=1}^{p} Q_{i}^{\prime}$ так, что для любого $i$ и любых $q_{0}, q_{0}^{\prime} \in Q_{i}^{\prime}$ выполняется равенство $\psi\left(q_{0}, \alpha\right)=\psi\left(q_{0}^{\prime}, \alpha\right)$, причем для разных $i, j$, если $q_{0} \in Q_{i}^{\prime}, q_{0}^{\prime} \in Q_{0}^{\prime}$, то $\psi\left(q_{0}, \alpha\right) \neq \psi\left(q_{0}^{\prime}, \alpha\right)$. Теперь из указанного листа направим $p$ ребер, помеченных словами $\psi\left(q_{i}, \alpha\right), q_{i} \in Q_{i}^{\prime}$. Концу $i$-го ребра припишем множество $\varphi\left(Q_{i}^{\prime}, \alpha\right)$. Очевидно, что начав с дерева, состоящего из единственной вершины с приписанным ей полным множеством состояний $Q$, мы, применяя описанную процедуру, построим некоторое дерево условного установочного эксперимента автомата $\mathfrak{U}$.

Рассмотрим произвольный путь от корня к листу в этом дереве. Пусть

$$
Q_{0}=Q, Q_{1}, \ldots, Q_{s}=\{q\}
$$

- последовательность множеств, приписанных вершинам пути, а $\alpha_{0}, \alpha_{1}, \ldots, \alpha_{s-1}$ - соответствующие слова, приписанные этим вершинам. Ясно, что $\left|Q_{0}\right|=n,\left|Q_{i}\right|>\left|Q_{i+1}\right|$, $\left|Q_{s}\right|=1$. Если $r_{j}$ - минимальное натуральное число такое, что $\left|\xi_{n_{r_{i}}}\right| \geqslant\left|Q_{i}\right|>\left|\xi_{n_{r_{i}+1}}\right|$, то согласно построению дерева условного установочного эксперимента можно утверждать, что $l\left(\alpha_{i}\right) \leqslant n_{r_{i}+1}$. Следовательно, длина указанной ветки дерева не превосходит $\sum_{i=1}^{s-1} n_{r_{i}+1}$. Отсюда следует, что длина максимальной ветви построенного дерева, ведущей от корня к листу, не превосходит $\sum_{j=1}^{k_{0}} n_{j}\left(\xi_{n_{j-1}}-\xi_{n_{j}}\right)$. Заметим теперь, что для всех $j$ выполняется неравенство $n_{j} \leqslant n-\xi_{n_{j-1}}+1$. В самом деле, введем обозначение $p=n-\xi_{n_{j-1}}+1$. Нетрудно понять, что $\xi_{p}<\xi_{n_{j-1}}$, следовательно, $n_{j} \leqslant p$, что и требовалось доказать. Также очевидно, что $n_{j} \leqslant h$. Положим $t_{j}=\min \left\{h, n-\xi_{n_{j-1}}+1\right\}$. Тогда доказываемое первенство мы можем переписать в виде

$$
l(\mathfrak{U}) \leqslant \sum_{j=1}^{k_{0}} t_{j}\left(\xi_{n_{j-1}}-\xi_{n_{j}}\right) .
$$

Пусть также $u_{j}=\max \left\{n+1-h, \xi_{n_{j-1}}\right\}$. Тогда

$$
\begin{aligned}
l(\mathfrak{U}) & \leqslant(n+1) \sum_{j=1}^{k_{0}}\left(\xi_{n_{j-1}}-\xi_{n_{j}}\right)-\sum_{j=1}^{k_{0}} u_{j}\left(\xi_{n_{j-1}}-\xi_{n_{j}}\right) \\
& =(n+1)(n-1)-\sum_{j=1}^{k_{0}} u_{j}\left(\xi_{n_{j-1}}-\xi_{n_{j}}\right) .
\end{aligned}
$$


Теперь из того, что $n_{k_{0}}=h$ нетрудно вывести, что

$$
\sum_{j=1}^{k_{0}} u_{j}\left(\xi_{n_{j-1}}-\xi_{n_{j}}\right) \geqslant n+(n-1)+\ldots+(n-h+2)+(n-h+1)(n-h) .
$$

Теперь окончательно получаем, что $L(\mathfrak{U}) \leqslant(1 / 2) h(2 n-1-h)$.

Докажем достижимость этой оценки. Рассмотрим автомат $\mathfrak{A}=(A, Q, B, \varphi, \psi)$, где $Q=\{1, \ldots, n\}, A=\{1, \ldots, n\}, B=\{0,1\}$, а функции переходов и выходов задаются следующим образом:

$$
\psi(i, j)= \begin{cases}0, & \text { если } i \neq 1, \text { либо } i=1, j \neq 1 \\ 1, & \text { если } i=1 \text { и } j=1\end{cases}
$$

при $i \geqslant h$

$$
\varphi(i, j)= \begin{cases}i & \text { при } j \neq i \\ h-1 & \text { при } j=i\end{cases}
$$

при $i=h-1$

$$
\varphi(i, j)= \begin{cases}j & \text { при } j \geqslant h, \\ i-1 & \text { при } j=h-1, \\ i & \text { в остальных случаях }\end{cases}
$$

при $i<h-1$

$$
\varphi(i, j)= \begin{cases}i-1 & \text { при } j=i \\ i+1 & \text { при } j=i+1 \\ i & \text { в остальных случаях }\end{cases}
$$

Нетрудно понять, что степень отличимости зтого автомата есть $h$. С другой стороны можно установить, что для этого автомата $l(\mathfrak{U})=(1 / 2) h(2 n-h-1)$, то есть на этом автомате достигается нижняя оценка из теоремы 7. Тем самым теорема 7 доказана.

Теперь сформулируем и докажем теорему о связи остаточной степени отличимости с длиной простого условного установочного эксперимента.

Теорема 8. Для автомата $\mathfrak{U}$ с $n$ состояниями при $h_{\mathrm{fin}}(\mathfrak{U})=h_{0}$ выполнено неравенство $L(\mathfrak{U}) \leqslant n h_{0}$.

Доказательство. Рассмотрим некоторое подмножество состояний автомата $Q_{0}$ и возьмем два состояния $q_{0}, q_{1}$ из этого подмножества. По определению найдется слово $\alpha$ длины, не превосходящей $h_{0}$, которое либо склеивает два этих состояния, либо различает их. Таким образом, мощность множества, полученного из $Q_{0}$ после подачи на вход автомата слова $\alpha$, как минимум на единицу меньше мощности множества $Q_{0}$. Следовательно, простой условный установочный эксперимент можно проводить, подавая последовательно на вход слова указанным способом. Очевидно, что длина такого эксперимента не превосходит $n h_{0}$. Отсюда $L(\mathfrak{U}) \leqslant n h_{0}$, что и требовалось доказать. 


\section{5. Теорема об условной степени отличимости}

Введем понятие энтропии разбиения. Пусть дано разбиение $T$ множества $Q$. Представим его в виде $T=\left\{Q_{1}, Q_{2}, \ldots, Q_{k}\right\}$. Мощность множества $Q_{i}$ обозначим $s_{i}, i=1, \ldots, k$. Величину

$$
\chi(T)=\sum_{1 \leqslant i \leqslant k} s_{i} \log \left(s_{i}\right)
$$

назовем энтропией разбиения $T$. Сформулируем и докажем следующее утверждение.

Предложение 1. Если $T$ - разбиение множества $Q, T^{\prime}$ - его подразбиение и $\operatorname{ord}\left(T, T^{\prime}\right) \geqslant M$, mогда $\chi(T)-\chi\left(T^{\prime}\right) \geqslant 2 M$.

Доказательство. Докажем следующее неравенство. Пусть $n \geqslant 2, x_{1}, \ldots, x_{k} \geqslant 1$, $x_{1} \geqslant x_{2}, x_{1} \geqslant x_{3}, \ldots, x_{1} \geqslant x_{k}, x_{1}+x_{2}+\ldots+x_{k}=n$. Тогда

$$
n \log n-x_{1} \log \left(x_{1}\right)-\ldots-x_{k} \log \left(x_{k}\right) \geqslant 2\left(n-x_{1}\right) .
$$

Доказательство этого неравенства проведем индукцией по $k$. При $k=2$ и $x_{1} \geqslant n-x_{1}$,

$$
n \log n-x_{1} \log \left(x_{1}\right)-\left(n-x_{1}\right) \log \left(n-x_{1}\right) \geqslant 2\left(n-x_{1}\right) \text {. }
$$

Это неравенство доказывается с применением стандартных приемов математического анализа. Сделаем теперь индуктивный переход. Объединим слагаемые $x_{1}$ и $x_{2}$. Тогда по индуктивному предположению справедливо неравенство

$$
n \log n-\left(x_{1}+x_{2}\right) \log \left(x_{1}+x_{2}\right)-x_{3} \log \left(x_{3}\right)-\ldots-x_{k} \log \left(x_{k}\right) \geqslant 2\left(n-\left(x_{1}+x_{2}\right)\right) .
$$

Учитывая теперь, что

$$
\left(x_{1}+x_{2}\right) \log \left(x_{1}+x_{2}\right)-x_{1} \log \left(x_{1}\right)-x_{2} \log \left(x_{2}\right) \geqslant 2\left(\left(x_{1}+x_{2}\right)-x_{1}\right),
$$

получим требуемое неравенство.

Представим теперь разбиение $T$ в виде $T=\left\{Q_{1}, \ldots, Q_{s}\right\}$. Пусть $Q_{i_{0}}, Q_{i_{1}}, \ldots, Q_{i_{p}}$ - все те элементы разбиения $T$, которые подразбиваются в разбиении $T^{\prime}$. Представим множество $Q_{i_{r}}$ в виде $Q_{i_{r}}=Q_{r_{1}}^{\prime} \cup Q_{r_{2}}^{\prime} \cup \ldots \cup Q_{r_{v}}^{\prime}$. Пусть $v_{0}$ - индекс максимальной по мощности компоненты $Q_{r_{v}}^{\prime}$. Тогда согласно доказанному неравенству убывание энтропии разбиения $T^{\prime}$ при подразбиении множества $Q_{i_{r}}$ не меньше, чем $2\left(\left|Q_{i_{r}}\right|-\left|Q_{r_{0}}\right|\right)$. Тогда учитывая определение порядка подразбиения относительно разбиения, нетрудно видеть, что суммарное убывание энтопии подразбиения $T^{\prime}$ не меньше, чем $2 \operatorname{ord}\left(T^{\prime}, T\right)$. По условию $\operatorname{ord}\left(T^{\prime}, T\right) \geqslant M$, поэтому $\chi(T)-\chi\left(T^{\prime}\right) \geqslant 2 M$, что и требовалось доказать.

Для автомата $\mathfrak{U}$ степени отличимости $h_{0}$ обозначим через $T_{k}, 0 \leqslant k \leqslant h_{0}$, разбиение, определяемое отношением $R_{k}$, и через $N(M)$ обозначим число разбиений $T_{k}$ таких, что $\operatorname{ord}\left(T_{k}, T_{k-1}\right) \geqslant M$. Докажем следующее утверждение.

Предложение 2. Для приведенного автомата $\mathfrak{X}$ с $n$ состояниями

$$
N(M) \leqslant(n \log (8 M)) / M \text {. }
$$


Доказательство. Рассмотрим цепочку подразбиений $T_{0}, T_{1}, T_{2}, \ldots, T_{s}$, где $T_{0}$ - разбиение, состоящее из всего $n$-элементного множества $Q$ и $T_{s}$ - разбиение, состоящее из одноэлементных множеств. Нетрудно видеть, что существует не более, чем $n /(2 M)$ переходов $T_{i} \rightarrow T_{i+1}$ таких, что хотя бы одно из множеств разбиения $T_{i}$ подразбивается таким образом, что одна из частей подразбиения имеет мощность большую, чем $2 M$. При этом, очевидно, можно считать, что все такие переходы находятся в начале цепочки. Оставшуюся часть цепочки можно удлинить до цепочки $T_{i_{1}+1}, T_{i_{2}+2}, \ldots, T_{i_{1}+r}$ так, что первые не более чем $r<n /(2 M)$ переходов устроены таким образом, что в разбиении $T_{i_{1}+r}$ все множества имеют мощность, не большую $4 M$, и те не более, чем $n /(2 M)$ множеств, которые имеют мощность большую, чем $2 M$, при дальнейшем дроблении могут дробиться таким образом, что та из частей, которая возникает при дроблении и не учитывается в сумме, определяющей порядок подразбиения, может быть не обязательно максимальной по мощности среди возникающих частей разбиения. Назовем такие множества особыми. Путем удвоения числа переходов мы далее можем преобразовать остаток цепочки таким образом, что разбиения таких особых множеств происходят на не более, чем две части. Переходов в полученной цепочке, при которых особые множества дробятся на две части и при этом в сумму, определяющую порядок подразбиения, входит большая из возникающих частей, очевидно не более, чем $n /(2 M) \log (4 M)$; все остальные переходы устроены стандартным образом, то есть порядок каждого подразбиения не меньше, чем $M$, и порядок подсчитывается обычным способом.

Очевидно, что $\chi\left(T_{i_{1}+r}\right) \leqslant n \log (4 M)$. Далее, очевидно, что энтропия - убывающая функция и в случае стандартных переходов, если $\operatorname{ord}\left(T_{k}, T_{k-1}\right) \geqslant M$, то $\chi\left(T_{k-1}\right)-\chi\left(T_{k}\right) \geqslant 2 M$ согласно предложению 1. Учитывая теперь, что по определению энтропия есть неотрицательная величина, получим, что

$$
N(M) \leqslant 2 \frac{n \log (4 M)}{2 M}+\frac{n}{2 M}+\frac{n}{2 M} \leqslant \frac{n \log (8 M)}{M} .
$$

Утверждение доказано.

Докажем наконец теорему об условной степени отличимости.

Теорема 9. Для любого приведенного автомата $\mathfrak{U}$ c n состояниями и произвольного $p \geqslant 1$

$$
h_{c}(\mathfrak{A}, p) \leqslant \frac{n \log (9 p)}{p} .
$$

Доказательство. Рассмотрим неприводимый автомат $\mathfrak{U}=(A, Q, B, \varphi, \psi)$ такой, что $|Q|=n$ и $h(\mathfrak{U})=h_{0}$. Рассмотрим семейство разбиений $T_{k}, 0 \leqslant k \leqslant h_{0}$, множества $Q$, индуцируемых отношениями $R_{k}$.

Пусть $\alpha=(\log (9 p)) / p$. Если $h_{0} \leqslant n \alpha$, то доказывать нечего. В противном случае подберем целые неотрицательные числа $s$ и $k$ таким образом, что $n \alpha-2 \leqslant 2 s \leqslant n \alpha$, $h_{0}-s k \leqslant s$. Очевидно, что это всегда можно сделать. Введем обозначение

$$
m_{i}=\min _{i s \leqslant j \leqslant(i+1) s} \operatorname{ord}\left(T_{j}, T_{j-1}\right), \quad i=0,1, \ldots, k .
$$

Соответствующий минимизирующий индекс обозначим $j_{i}$. Таким образом, $\operatorname{ord}\left(T_{j_{i}}, T_{j_{i}-1}\right)=m_{i}$. Рассмотрим последовательность $\gamma=\left\{j_{0}, \ldots, j_{k-1}\right\}$. По построению $\operatorname{disp}(\gamma) \leqslant n \alpha$. Если теперь доказать, что порядок дробления автомата $\mathfrak{A}$ относительно последовательности $\gamma$ не превосходит $p$, то согласно определению условной степени отличимости автомата, это будет означать, что $h_{c}(\mathscr{U}, p) \leqslant(n \log (9 p)) / p$. 
Порядок дробления автомата $\mathfrak{U}$ относительно последовательности $\gamma$ равен $S=m_{0}+m_{1}+\ldots+m_{k-1}$. Проверим, что последняя сумма не превосходит $p$. В самом деле обозначим $k_{r}$ - число членов указанной суммы, равных $r$. Докажем, что $k_{r}=0$ при $r>(n \log (8 p) / s$. В самом деле, предположим, что это не так. Это означает, что найдется $j, 0 \leqslant j \leqslant k-1$, такое, что $m_{j}=r$. Тогда, применяя предложение 2 , нетрудно заключить, что $s \leqslant(n \log (8 p) / r$, то есть $s<s$. Получаем противоречие.

Положим $(n \log (8 p)) / s=R$. Тогда $S=\sum_{1 \leqslant r \leqslant R} r k_{r}$. Введем обозначение $K_{r}=\sum_{r \leqslant i \leqslant R} k_{i}$. Тогда, применяя к сумме $S$ преобразование Абеля, получим, что $S=\sum_{1 \leqslant r \leqslant R} K_{r}$. Оценим, наконец, слагаемое $K_{r}$. Нетрудно убедиться, что в условиях предложения $2 N(r)>K_{r} s$. Поэтому согласно этому предложению $N(r) \leqslant(n \log (8 p)) / r$. Таким образом, $s K_{r} \leqslant(n \log (8 p)) / r$, или $K_{r} \leqslant R / r$. Следовательно, $S \leqslant \sum_{1 \leqslant r \leqslant R} R / r \leqslant R \log R$. Вспоминая, что $R=(n \log (8 p)) / s$ и $s \geqslant n \alpha-2$, после несложных преобразований, получаем, что $R \log R \leqslant p$, откуда $S \leqslant p$, что заканчивает доказательство теоремы.

\section{6. Доказательство теоремы 1}

Докажем следующее более сильное утверждение, из которого вытекает теорема 1.

Предложение 3. Справедливы соотношения

$$
\begin{aligned}
& g(n, p) \leqslant h(n, m, p) \leqslant \min \{(n m \log (9 p)) / p+1, n-1\} \\
& g(n, p) \leqslant h^{\prime}(n, p) \leqslant \min \{(n \log (9 p)) / p, n-1\}
\end{aligned}
$$

Доказательство. Из теорем 5, 6 и 9 несложно вывести верхние оценки предложения 3. Для получения нижних оценок рассмотрим автомат $\mathfrak{c}=(A, Q, B, \varphi, \psi)$, где $Q=\{1, \ldots, n\}, A=\{1, \ldots, n\}, B=\{0,1\}$, а функции переходов и выходов задаются следующим образом:

$$
\psi(i, j)= \begin{cases}0, & \text { если } i \neq 1, \text { либо } i=1, j \neq 1 \\ 1, & \text { если } i=1 \text { и } j=1\end{cases}
$$

при $i \geqslant 2$

$$
\varphi(i, j)= \begin{cases}i-1 & \text { при } j=i \\ i+1 & \text { при } j=i+1 \\ i & \text { в остальных случаях }\end{cases}
$$

при $i=1$

$$
\varphi(i, j)= \begin{cases}1 & \text { при } j=1 \\ 2 & \text { при } j=2 \\ i & \text { в остальных случаях }\end{cases}
$$

Нетрудно понять, что на этом автомате достигаются нижние оценки предложения 3. Предложение доказано. 


\section{7. Доказательство теоремы 2}

Докажем следующее более сильное утверждение, из которого вытекает теорема 2.

Предложение 4. Справедливы соотночения

$$
\begin{aligned}
& g(n, p) \leqslant h_{i d}(n, p) \leqslant g(n, p)+1, \\
& g(n, p) \leqslant h_{i d}^{\prime}(n, p) \leqslant g(n, p)+1 .
\end{aligned}
$$

Нижние оценки этого утверждения уже доказаны в предыдущем параграфе, так как, очевидно, что автомат, на котором достигалась нижняя оценка в общем случае является автоматом-идентификатором. Для доказательства верхних оценок нам потребуется доказать одну лемму об ориентированных деревьях.

Лемма 1. Для ориентированного к корню $s_{0}$ дерева $T$ c $n$ вершинами и натурального $p \geqslant 1$ найдется $r$ вершин дерева $s_{1}, \ldots, s_{r}, r \leqslant p$, таких, что множество $S$ вериин дерева разбивается на $r+1$ непересекаючуюся часть $S_{0}, \ldots, S_{r}$ так, что $s_{i} \in S_{i}$ и для любого $s \in S_{i}, i=1, \ldots, r$, найдется ориентированный путь, соединяющий $s$ c $s_{i}$ длины, не большей $g(n, p)$.

Доказательство. Мы несколько видоизменим утверждение леммы и будем в добавление к требуемым свойствам еще требовать выполнение свойства, которое назовем свойством $A$, состоящего в том, что если при некотором разбиении в случае, когда $n-1$ не делится нацело на $p+1$, для некоторой вершины $s_{\text {out }}$ справедливо включение $s_{\text {out }} \in S_{i}$ и $\operatorname{dist}\left(s_{\max }, s_{i}\right)=[(n-1) /(p+1)]+1$, где $\operatorname{dist}(a, b)$ обозначает длину кратчайшего ориентированного пути в дереве, ведущего от вершины $a$ к вершине $b$, то можно изменить это разбиение с сохранением всех свойств таким образом, что в новом разбиении, если $s_{\text {out }} \in S_{i}$, то $\operatorname{dist}\left(s_{\text {out }}, s_{i}\right)=[(n-1) /(p+1)]$.

Доказательство проведем индукцией по $p$. Сначала проверим утверждение леммы при $p=1$. В свою очередь указанное утверждение будем доказывать индукцией по числу вершин дерева $n$. При малых значениях $n$ утверждение очевидно. Пусть оно доказано для $n=n_{0}$. Докажем его при $n=n_{0}+1$. Доказательство проведем индукщией по параметру дерева $\ell(T)=n-1-\operatorname{dist}\left(s_{\max }, s_{0}\right)$, то есть по разности значения величины $\operatorname{dist}\left(s_{\max }, s_{0}\right)$ и своего максимально возможного значения $n-1$. В случае, когда $\ell(T)=0$, как нетрудно понять, дерево представляет собой простую ориентированную цепь, ведущую от $s_{\max } \kappa s_{0}$. В этом случае утверждение очевидно. Пусть утверждение доказано при $\ell(T)=\ell_{0}$. Проверим его при $\ell(T)=\ell_{0}+1$. Для этого рассмотрим вершину $s_{\max }$. Возможны два случая. В первом случае найдется вершина $s_{\max }^{\prime} \neq s_{\max }$ такая, что $\operatorname{dist}\left(s_{\max }^{\prime}, s_{0}\right)=\operatorname{dist}\left(s_{\max }, s_{0}\right)$. Во втором случае не существует вершины $s$ в дереве $T$ такой, что $\operatorname{dist}\left(s, s_{0}\right)=\operatorname{dist}\left(s_{\max }, s_{0}\right)$.

Рассмотрим первый случай. Преобразуем дерево $T$ следующим образом: введем дополнительную вершину $s^{\prime}$ и соединим ее с вершиной $s_{\max }^{\prime}$ ориентированным от вершины $s^{\prime}$ ребром, а вершину $s_{\max }$ удалим вместе с исходящим из нее ребром. Для полученного дерева $T^{\prime}$ справедливо равенство $\ell\left(T^{\prime}\right)=\ell_{0}$, и следовательно, к нему можно применить предположение индукщии и разбить вершины дерева $T^{\prime}$ на один или два класса с выполнением свойств из формулировки леммы. Предположим, что в дереве $T$ вершина $s_{\max }$ соединялась с вершиной $s^{\prime \prime}$. Если при разбиении дерева $T^{\prime}$ вершины $s^{\prime}$ и $s^{\prime \prime}$ попали в один класс, то разбиение дерева $T$ с требуемыми свойствами получается из разбиения дерева $T^{\prime}$ добавлением в класс вершин $s^{\prime}$ и $s^{\prime \prime}$ вершины $s_{\max }$, в противном случае искомое разбиение дерева $T$ получается из разбиения дерева $T^{\prime}$ добавлением $s_{\max }$ в класс вершины $s^{\prime \prime}$ в случае, если $\operatorname{dist}\left(s^{\prime \prime}, s_{i_{0}}\right) \leqslant[(n-1) / 2]$, где $s_{i_{0}}$ - вершина, участвующая в определении 
разбиения дерева из условия леммы такая, что $s^{\prime \prime} \in S_{i_{0}}$, в противном случае требуется некоторая очевидная модификация разбиения дерева $T^{\prime}$.

Во втором случае рассмотрим вершину $s^{\prime}$, смежную с $s_{\max }$, а также дерево $T^{\prime}$, получающееся из дерева $T$ путем отбрасывания вершины $s_{\max }$. Это дерево содержит $n_{0}$ вершин и к нему можно применить предположение индукции. Полагая при этом $s_{\text {out }}=s^{\prime}$, получаем, что добавляя вершину $s_{\max }$ к той части разбиения дерева $T^{\prime}$, которое содержит $s^{\prime}$, получим разбиение дерева $T$ с требуемыми свойствами. При $p=1$ нетрудно также убедиться в справедливости отмеченного свойства $A$ полученного разбиения дерева $T$. Таким образом, заканчивается рассмотрение случая $p=1$.

Пусть теперь утверждение леммы доказано при $p=p_{0}$. Проверим его справедливость при $p=p_{0}+1$. Доказательство вновь будем вести индукщией по $n$. Предположим, что утверждение доказано при $n=n_{0}$, и докажем его для $n=n_{0}+1$. Здесь, как и в случае $p=1$, рассуждение также ведем индукщией по $\ell(T)$. В случае $\ell(T)=0$ дерево превращается в простую цепь и утверждение очевидно. Пусть мы проверили выполнимость всех требований леммы и выполнимость свойства $A$ при $\ell(T)=\ell_{0}$. Проверим все эти свойства в случае $\ell(T)=\ell_{0}+1$. Здесь рассуждаем, как и в случае $p=1$. Пусть $s_{\max }$ одна из вершин, удаленных на максимальное расстояние от корня дерева. Возможны два случая.

В первом случае найдется вершина $s_{\max }^{\prime} \mathrm{c} \operatorname{dist}\left(s_{\max }^{\prime}, s_{0}\right)=\operatorname{dist}\left(s_{\max }, s_{0}\right)$.

Во втором случае не существует вершины $s \operatorname{cdist}\left(s, s_{0}\right)=\operatorname{dist}\left(s_{\max }, s_{0}\right)$.

В первом случае строим дерево $T^{\prime}$ так же, как в случае $p=1$. Применяя индукцию, строим разбиение дерева $T^{\prime}$, обладающее всеми свойствами, указанными в лемме. В случае, когда вершины $s^{\prime}$ и $s^{\prime \prime}$ попадут в один класс разбиения, требуемое разбиение дерева $T$ получается добавлением в указанный класс вершины $s_{\max }$. В противном случае полагаем $s_{\text {out }}=s^{\prime \prime}$ и строим новое разбиение дерева $T^{\prime}$ с учетом свойства $A$ и добавляем к той части нового разбиения, которое содержит вершину $s^{\prime \prime}$, вершину $s_{\max }$. Нетрудно проверить, что таким образом мы строим требуемое разбиение дерева $T$.

Во втором случае, как и при $p=1$, обозначаем $s^{\prime}$ вершину, смежную с $s_{\max }$, и рассматриваем дерево $T^{\prime}$, получающееся из дерева $T$ путем удаления вершины $s_{\max }$. Оно содержит $n_{0}$ вершин и к нему можно применить предположение индукции. Полагая при этом $s_{\text {out }}=s^{\prime}$, мы строим разбиение дерева $T^{\prime}$, которое при добавлении к той его части, которая содержит $s^{\prime}$, вершины $s_{\max }$, становится требуемым разбиением дерева $T$.

Наконец, проверим выполнимость свойства $A$. Предположим, что $n-1$ не делится нацело на $p+1$. Пусть в построенном разбиении $\operatorname{dist}\left(s_{\text {out }}, s_{i}\right)=[(n-1) /(p+1)]+1$. Рассмотрим ориентированный путь из $s_{\text {out }}$ В $s_{i}$. Рассмотрим предпоследнюю вершину $s^{\prime \prime}$ этого пути. Удалим из дерева $T$ поддерево, растущее из вершины $s^{\prime \prime}$, которое мы обозначим $T^{\prime \prime}$. Это поддерево содержит не менее $[(n-1) /(p+1)]+1$ вершин. К оставшемуся поддереву применим лемму с $p=p_{0}$. Добавив теперь к получившемуся разбиению еще одну часть $S_{i}=T^{\prime \prime}$ с $s_{i}=s^{\prime \prime}$, получим разбиение дерева $T$ со всеми требуемыми свойствами, для которого теперь $\operatorname{dist}\left(s_{\text {out }}, s_{i}\right)=[(n-1) /(p+1)]$, что и требовалось доказать.

Лемма полностью доказана.

Перейдем к доказательству предложения 4.

Рассмотрим автомат-идентификатор $\mathfrak{A}$ с $n$ состояниями. Выделим в нем состояние $q_{0}$, в котором на выходе может вырабатываться символ, отличный от всех выходных символов, вырабатываемых на выходе остальных состояний. Такое состояние существует и единственно по определению. В диаграмме переходов автомата $\mathfrak{U}$ можно выделить подграф, являющийся ориентированным к корню деревом с корнем в $q_{0}$. Применяя к этому 
дереву лемму 1 , выделяем не более $p$ вершин из $s_{i}, \ldots, s_{r}$, для которых выполняются свойства из леммы 1 . Для доказательства первого неравенства теоремы 2 достаточно теперь зафиксировать некоторый входной символ $a$ автомата $\mathfrak{U}$ и положить $\varphi^{\prime}\left(s_{i}, a\right)=q_{0}$ для любого $i, 1 \leqslant i \leqslant r$. Для доказательства второго неравенства достаточно рассмотреть $r$ различных выходных символов $b_{1}, \ldots, b_{r}$, не входящих в выходной алфавит автомата $\mathfrak{A}$, рассмотреть некоторый входной символ $a$ автомата $\mathfrak{A}$ и положить $\psi^{\prime}\left(s_{i}, a\right)=b_{i}$ для любого $i, 1 \leqslant i \leqslant r$. Нетрудно теперь понять, что верхние оценки для функций $h_{\text {fin }}$ и $h$ у соответствующих автоматов совпадают с верхними оценками предложения 4. Утверждение, а вместе с ним и теорема 2, доказаны.

\section{8. Доказательство теорем 3 и 4}

Докажем более сильные утверждения, из которых вытекают теоремы 3 и 4.

Предложение 5. Справедливы соотношения

$$
\begin{gathered}
(p+1) g(n, p)(g(n, p)-1) / 2 \leqslant L(n, n, p) \frac{n^{2}}{4(p+1)^{2}} \leqslant L(n, 2, p), \\
L(n, m, p) \leqslant \min \left\{\frac{n^{2} m \log (9 p)}{p}+1, \frac{n(n-1)}{2}\right\}, \\
(p+1) g(n, p)(g(n, p)-1) / 2 \leqslant L^{\prime}(n, p) \leqslant \min \left\{\frac{n^{2} \log (9 p)}{p}, \frac{n(n-1)}{2}\right\} .
\end{gathered}
$$

Предложение 6. Справедливы соотношения

$$
\begin{aligned}
& (p+1) g(n, p)(g(n, p)-1) / 2 \leqslant L_{\text {id }}(n, p) \leqslant \min \left\{n g(n, p), \frac{n(n-1)}{2}\right\}, \\
& (p+1) g(n, p)(g(n, p)-1) / 2 \leqslant L_{\text {id }}^{\prime}(n, p) \leqslant \min \left\{\frac{n(2 n-g(n, p))}{2}, \frac{n(n-1)}{2}\right\} .
\end{aligned}
$$

Нижние оценки из предложений 5 и 6, за исключением оценки

$$
\frac{n^{2}}{4(p+1)^{2}} \leqslant L(n, 2, p),
$$

достигаются на автомате $\mathfrak{C}$, описанном в разделе 6 . Доказательство этого является несложным видоизменение доказательства из [3]. Комбинируя результаты теорем 1 и 9, получаем верхние оценки предложения 5 , а комбинируя результаты теорем 2 и 9, а также 2 и 8 , получаем верхние оценки предложения 6.

Докажем теперь оценку $n^{2} /\left(4(p+1)^{2}\right) \leqslant L(n, 2, p)$. Для этого рассмотрим автомат $\mathfrak{U}=(A, Q, B, \varphi, \psi)$ с $|A|=2,|B|=p+2,|Q|=n$. Положим $n=(p+1) 2 k$.

Случай, когда $n$ не делится нацело на $2(p+1)$, рассматривается аналогично. Состояния множества $Q$ разобьем на непересекающиеся группы состояний $Q=Q_{1} \cup Q_{2} \cup \ldots \cup Q_{p+1}$.

Положим также $B=\{0,1,2, \ldots, p+1\}$. Для всякого $i, 1 \leqslant i \leqslant p+1$, полагаем $Q_{i}=\left\{q_{i}^{1}, q_{i}^{2}, \ldots q_{i}^{2 k}\right\}$ и определяем функции переходов и выходов следующим образом: $\varphi\left(q_{i}^{j}, 0\right)=q_{i}^{j-1}$, если $j>1 ; \varphi\left(q_{i}^{1}, 0\right)=q_{i}^{k} ; \varphi\left(q_{i}^{j}, 1\right)=q_{i}^{j}$, если $j>k ; \varphi\left(q_{i}^{j}, 1\right)=q_{i}^{j+k}$, если $j \leqslant k ; \psi\left(q_{i}^{j}, 0\right)=0 ; \psi\left(q_{i}^{j}, 1\right)=0$, если $j \neq 1 ; \psi\left(q_{i}^{1}, 1\right)=i$. 
Нетрудным видоизменением доказательства теоремы 2 из [2], можно показать, что

$$
L(\mathfrak{U}, p) \geqslant \frac{n^{2}}{4(p+1)^{2}},
$$

что заканчивает доказательство указанной оценки.

В заключение автор приносит искреннюю благодарность В. Б. Кудрявцеву и А. С. Подколзину за поддержку и помощь в работе.

\section{Список литературы}

1. Кудрявцев В. Б., Алешин С. В., Подколзин А. С., Введепие в теорию автоматов. Наука, Москва, 1985.

2. Кирнасов А. Е., О длине простого условного установочного эксперимента. Иителлектуальные системы (2000) 5, №1-4, 199-212.

3. Козловский В. А., О распознавании автомата относительно локально порожденного класса. Докл. AH CCCP (1981) 258, №5, 1047-1049.

Статъя поступила 16.12.2004. 